\title{
BACH2 expression associates with survival in triple negative breast cancer.
}

Shahan Mamoor, $\mathrm{MS}^{1}$

${ }^{1}$ shahanmamoor@gmail.com

East Islip, NY USA

We mined published microarray data (1) to understand the most significant gene expression differences in the tumors of triple negative breast cancer patients based on survival following treatment: dead or alive. We observed significant transcriptome-wide differential expression of BTB and CNC homology 1, basic leucine zipper transcription factor 2, encoded by $\mathrm{BACH} 2$ when comparing the primary tumors of triple negative breast cancer patients dead or alive. Importantly, BACH2 expression was correlated with overall survival in patients with breast cancer. $\mathrm{BACH} 2$ may be of relevance as a biomarker or as a molecule of interest in understanding the etiology or progression of triple negative breast cancer.

Keywords: triple negative breast cancer, TNBC, systems biology of breast cancer, targeted therapeutics in breast cancer, BACH2, BTB and $\mathrm{CNC}$ homology 1, basic leucine zipper transcription factor 2 . 
Triple negative breast cancer is defined by lack of expression of the receptors for the hormones progesterone and estrogen (PR negative and ER negative), as well as for the human epidermal growth factor receptor 2 (HER2 negative) $(2,3)$. Though not completely identical, triple negative breast cancer shares significant overlap with the basal or basal-like molecular subtype of human breast cancer (3). TNBC is more frequently diagnosed in women of African descent (black women) for reasons not understood, and their disease course is similarly more aggressive than in white women with triple negative breast cancer $(2,3)$. To understand the transcriptional biology of triple negative breast cancer in an unbiased fashion and at the systems level, we mined published microarray data paired with patient survival data (1), performing comparative transcriptome analysis based on survival at time of data collection: dead or alive. We present here a finding from the results of this analysis.

\section{Methods}

We used dataset GSE142102 (1) for this differential gene expression analysis in conjunction with GEO2R. GSE142102 was generated using Affymetrix Human Gene 2.1 ST Array technology; in this analysis, we used data from $n=87$ tumors from TNBC patients that were dead at time of analysis and $n=123$ tumors from TNBC patients that were alive at time of analysis. The Benjamini and Hochberg method of $\mathrm{p}$-value adjustment was used for ranking of differential expression but raw $p$-values were used to assess statistical significance of global differential expression. Log-transformation of data was auto-detected, and the NCBI generated category of platform annotation was used. A statistical test was performed to evaluate whether $\mathrm{BACH} 2$ expression was significantly different between the primary tumors of TNBC patients that were dead or alive using a two-tailed t-test. For Kaplan-Meier survival analysis, we used the Kaplan-Meier plotter tool (4) for correlation of BACH2 mRNA expression levels with overall survival (OS) in $n=1090$ patients with breast cancer.

\section{Results}

We performed comparative transcriptome analysis using published microarray data (1) to understand in an unbiased fashion the most significant gene expression differences in the primary tumors of patients with triple negative breast cancer based on survival outcomes.

$\underline{\mathrm{BACH} 2}$ is differentially expressed in triple negative breast cancer and negatively correlates with survival.

When comparing primary tumors of 87 live patients with triple negative breast cancer with that of 123 dead TNBC patients we identified BTB and CNC homology 1, basic leucine zipper transcription factor 2, $\mathrm{BACH}$, as among the most significant transcriptional differences based on survival in human triple negative breast cancer. When ranking each of the transcripts whose expression was measured by microarray based on significance of difference in expression between the tumors of patients dead and alive, BACH2 ranked 213 out of 53617 total transcripts, equivalent to $99.6 \%$ differential expression (Chart 1). BACH2 differential expression in the tumors of patients based on survival outcomes was statistically significant (Figure $1 ; p=0.00523251$ ).

$\mathrm{BACH} 2$ is expressed at significantly higher levels in the breast tumors of TNBC patients when comparing based on survival.

We obtained exact mRNA expression levels for $\mathrm{BACH} 2$ from breast tumors of TNBC patients dead 
and alive to understand the magnitude and direction of $\mathrm{BACH} 2$ expression change based on survival outcomes. BACH2 was expressed at higher levels in tumors of TNBC patients alive as compared to that of TNBC patients dead (Figure 1). Increased expression of BACH2 in primary breast tumors was statistically significant (Figure 1: $p=0.0049$ ). $\mathrm{BACH} 2$ was expressed at $-0.23 \pm 0.88$ arbitrary units (AU) in the primary tumors of TNBC patients alive, while it was expressed at $0.18 \pm 1.04 \mathrm{AU}$ in the primary tumors of TNBC patients dead.

$\underline{\mathrm{BACH} 2 \text { expression correlates with overall survival in human breast cancer. }}$

We referenced a bioinformatics tool containing Kaplan-Meier survival data of human breast cancer patients to understand more thoroughly whether a relationship existed between BACH2 primary tumor expression and patient survival in human breast cancer. We found that $\mathrm{BACH} 2$ expression was correlated with overall survival in patients with breast cancer; this correlation approached statistical significance (Figure 2; log rank $p$-value: 0.054 for overall survival, hazard ratio: 0.73 (0.53-1.01) (Fig. 2)). BACH2 mRNA levels were a positive prognostic indicator in basal subtype breast cancer patients, though median OS data do not reflect this trend. Median OS was 131.37 months for patients with low tumor expression of BACH2 as compared to 130.87 months for patients with high tumor expression of BACH2.

Thus, we found through unbiased comparative transcriptome analysis that $\mathrm{BACH} 2$ was among the genes whose expression was most significantly different in the primary tumors of triple negative breast cancer patients alive when compared to that of TNBC patients dead, that BACH2 was expressed at significantly higher levels in the primary tumors of TNBC patients alive as compared to TNBC patients dead, and that $\mathrm{BACH} 2$ expression was positively correlated with overall survival in patients with breast cancer.

\section{Discussion}

It is not understood why women of African descent (black women) are diagnosed with triple negative breast cancer more frequently than white women, and why this disease is more aggressive in black women. BACH2 is important for antibody class-switching in B-cells (5) and for control of inflammation by influencing lineage-specification in T-cells (6). BACH2 may be of relevance as a biomarker in identifying patients who are more likely to survive, perhaps as part of a panel of genes used for prognostic stratification, and the molecule itself may be important to the biology of triple negative breast cancer etiology or more likely its progression. Blind systems-level analyses, like this, can help shed light on the fundamental molecular characteristics of a disease whose racial propensities demand further explanation. 


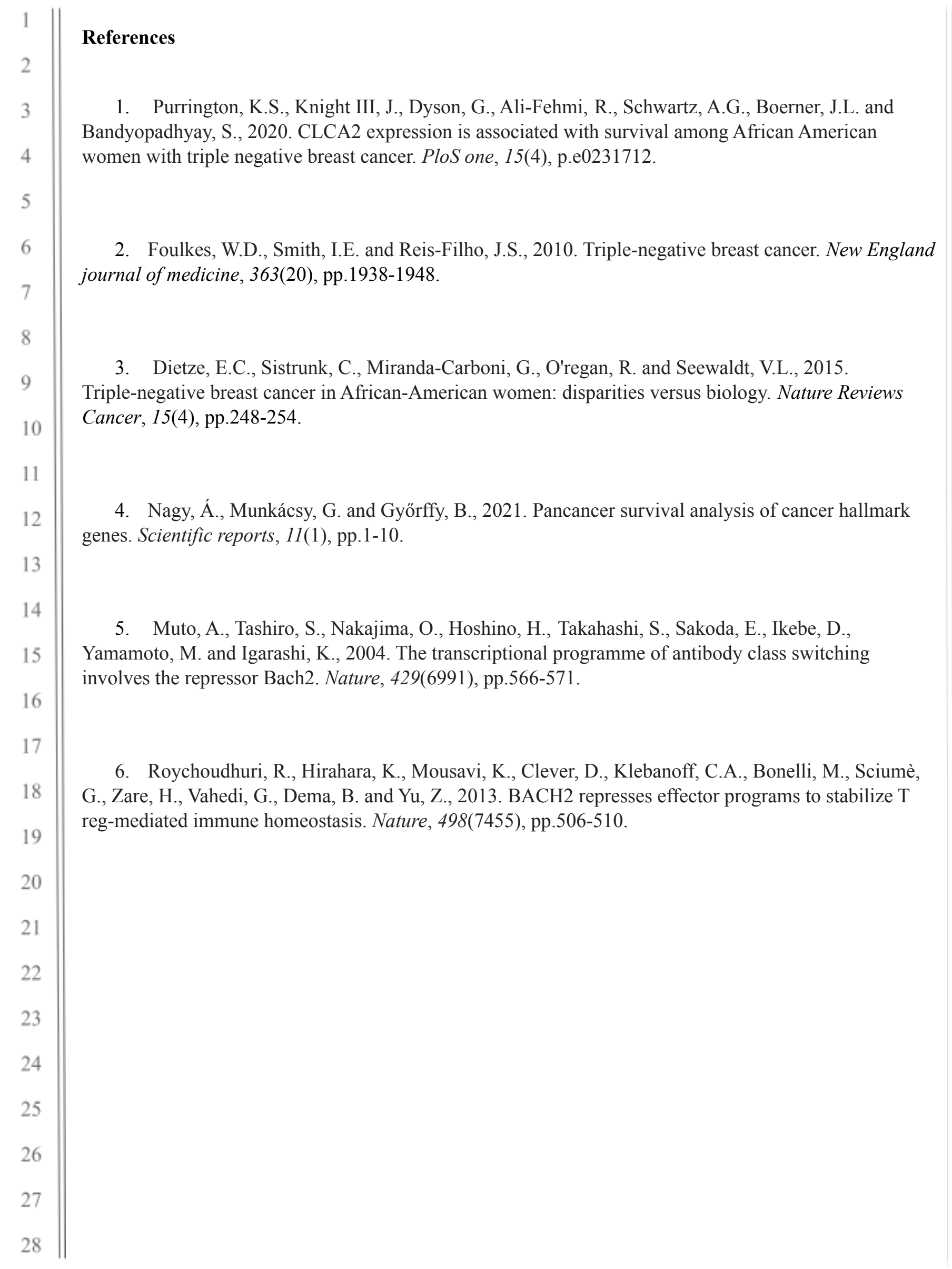




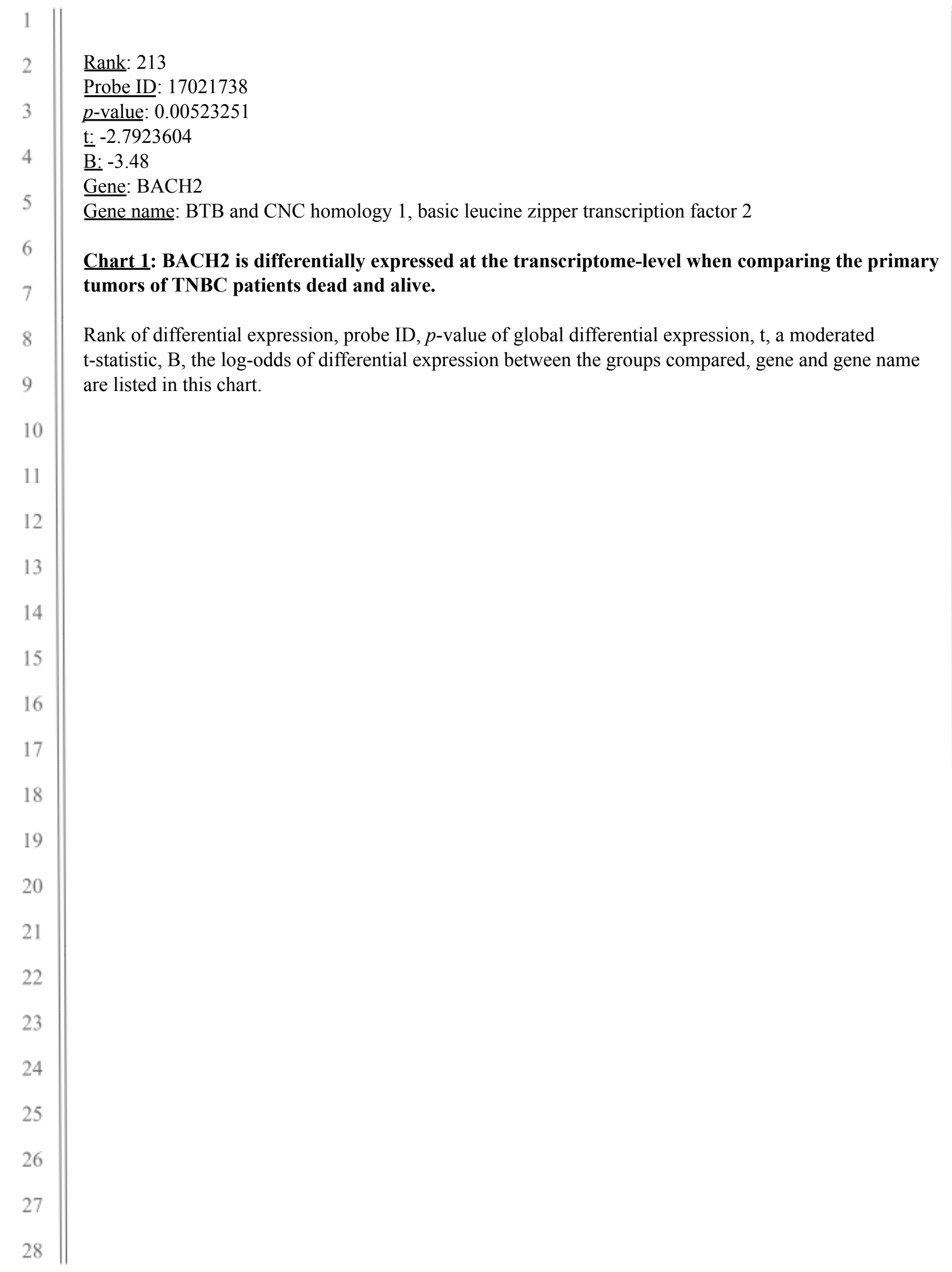




\section{BACH2}

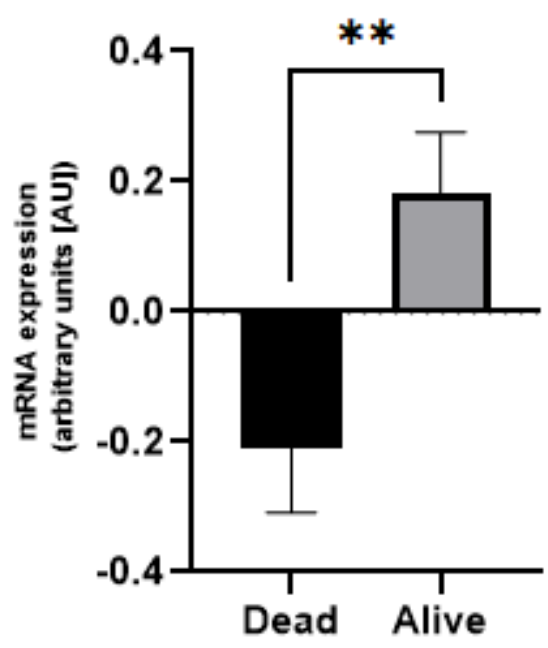

Figure 1: BACH2 is expressed at significantly higher levels in the primary tumors of TNBC patients alive as compared to the primary tumors of TNBC patients dead.

The mRNA expression level of BACH2 in the primary tumors of TNBC patients dead (left) and in primary tumors of TNBC patients alive (right) is graphically depicted with the result of a statistical test evaluating significance of difference in $\mathrm{BACH} 2$ expression between the primary tumors of TNBC patients dead and alive, a $p$-value, listed here: $p=0.0049$. 


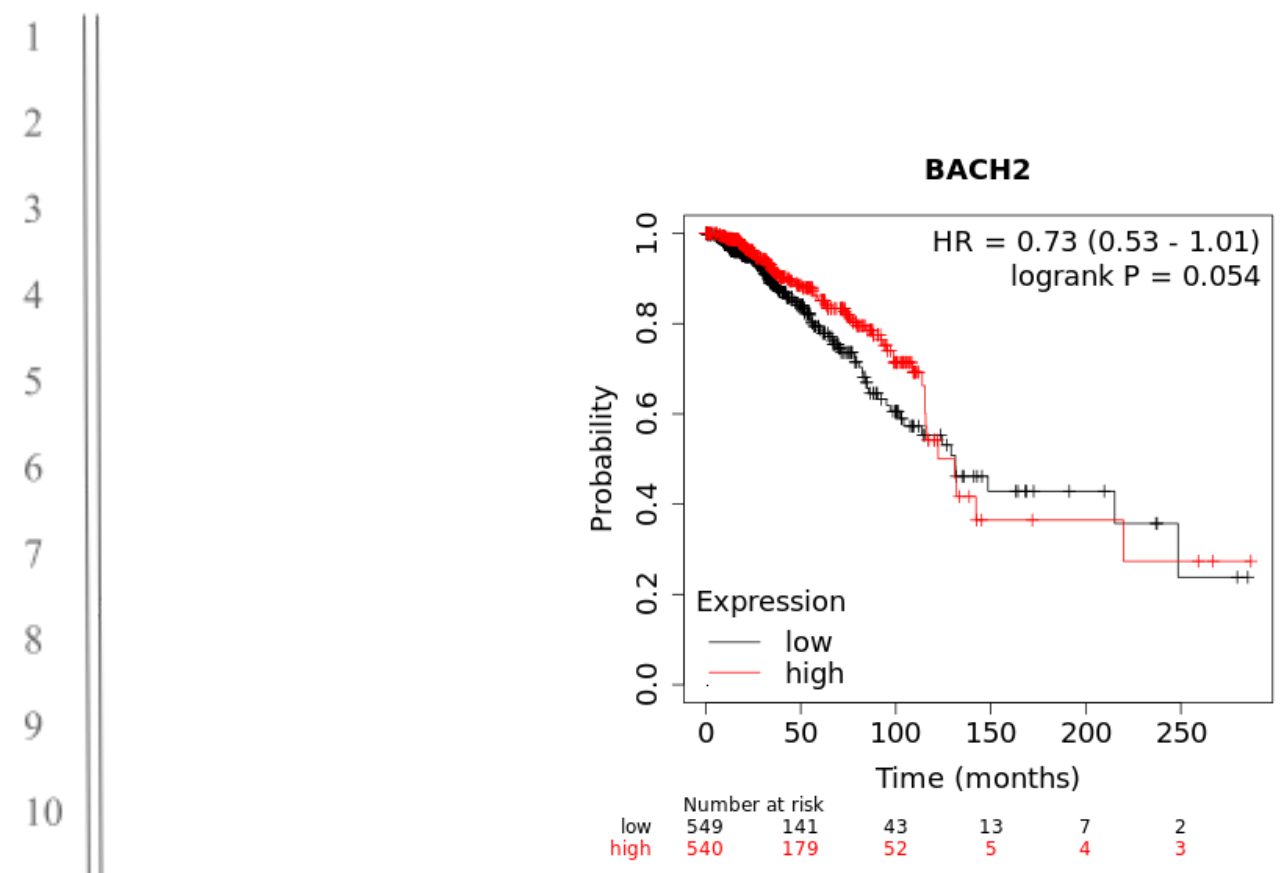

Figure 2: BACH2 expression correlates with overall survival in human breast cancer.

Depicted in this Kaplan-Meier plot is the probability of overall survival (OS) for $n=1090$ breast cancer patients based on low or high expression of $\mathrm{BACH} 2$ in patient primary tumors. The log rank p-value denoting statistical significance of difference in recurrence-free survival when comparing the two groups, as well as hazard ratio for this comparison is listed above. Listed below is the number of patients at risk (number of patients alive) per interval, after stratification based on $\mathrm{BACH} 2$ expression; in the first interval, number at risk is number of patients alive; in each subsequent interval, number at risk is the number at risk less those who have expired or are censored. 


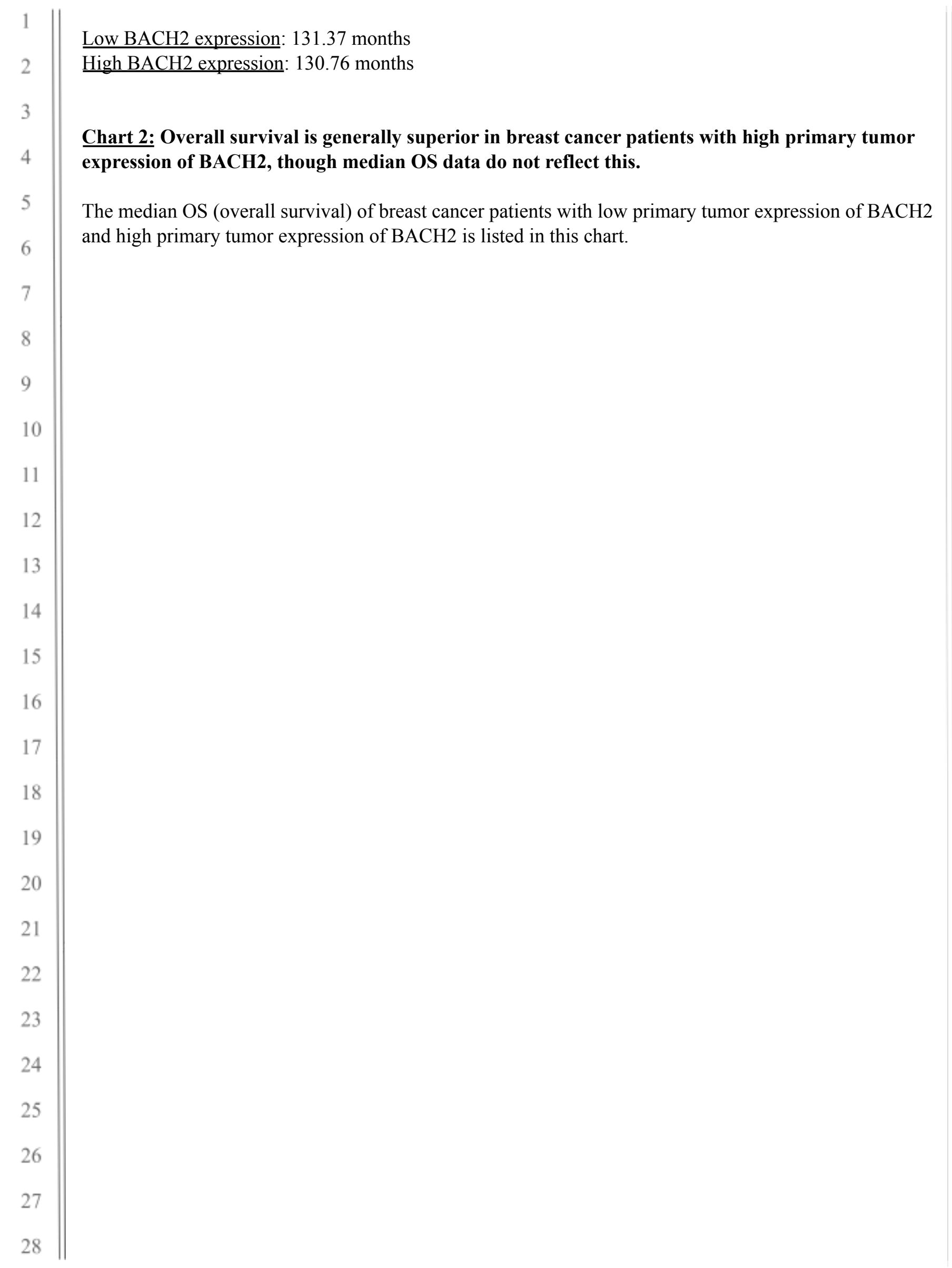

